Supporting Information

\title{
Accumulation, distribution and toxicity of arsenate associated with titanium dioxide nanoparticles in Daphnia magna
}

Mengting Lii ${ }^{1}$, Zhuanxi Luo ${ }^{1,2^{*}}$, Yamen Yan $^{1}$, Zhenhong Wang ${ }^{1}$, Qiaoqiao Chi ${ }^{1}$, Changzhou Yan ${ }^{1, *}$, Baoshan Xing $^{2}$

1, Key Laboratory of Urban Environment and Health, Institute of Urban Environment, Chinese Academy of Sciences, Xiamen 361021, China

2, Stockbridge School of Agriculture, University of Massachusetts, Amherst, Massachusetts 01003, United States

Number of Pages: 6

Number of Tables: 1 (Table S1)

Number of Figures: 3 (Figure S1 to Figure S3) 


\section{Supplementary Information for Accumulation Analysis of nano-TiO2 and $A s$.}

For particle content (body burden of arsenic and titanium in daphnia) analyses, sampled daphnia were treated following a modified method of Nathalie Adam et al. (Adam et al. 2014). They were washed for a few seconds in pure water to wash off the surrounding exposure medium, then washed in $5 \mathrm{mM} \mathrm{Na}_{2}$ EDTA for 1 min to remove externally bound nanoparticles and aggregates. Then we used $0.1 \mathrm{M}$ potassium phosphate buffer ( $\mathrm{pH} 7.0)$ to remove arsenic. After quickly washing in ultrapure water to remove the EDTA and potassium phosphate buffer, we put the daphnids samples into bullet vials. All vials were placed in a dry oven at $60^{\circ} \mathrm{C}$ for at least $48 \mathrm{~h}$ until a constant dry weight. To each vial, containing dried daphnids, $50 \mu \mathrm{L} \mathrm{HNO}_{3}(69 \%)$ and (after 12 h) $50 \mu \mathrm{L} \mathrm{HF(40 \% )} \mathrm{was} \mathrm{added.} \mathrm{The} \mathrm{daphnids} \mathrm{were} \mathrm{dissolved} 4 \mathrm{~h}$ later by microwave digestion $(4 \min 100 \mathrm{~W}, 3 \min 180 \mathrm{~W}, 2 \min 180 \mathrm{~W}, 2 \min 300 \mathrm{~W}, 2 \mathrm{~min}$ $300 \mathrm{~W}, 2$ min $450 \mathrm{~W}$ ), after which the samples were diluted to $1-2 \% \mathrm{HNO}_{3}$. The As and Ti concentration of the daphnids was measured by ICP-MS (Thermo Scientific Element 2 XR). For water samples, they were directly evaporated to dryness and then measured following the protocol described above. The Agilent 7500a ICP-MS was used at the conditions described in Table 1. Internal standards were rhodium and indium (1000 $\mu \mathrm{g} \mathrm{mL}^{-1}$, SCP Science) and the monitored masses were $75 \mathrm{As}, 115 \mathrm{In}$, $103 \mathrm{Rh}$ and $82 \mathrm{Se}$ for equation correction of interferences. The deviation of the instrument was below 2\% during the analysis and the limit of detection of the instrument for arsenic was calculated as $0.2 \mu \mathrm{g} \mathrm{L} \mathrm{L}^{-1}$. For quality control, two standard solutions of 5 and $20 \mu \mathrm{g} \mathrm{L}^{-1}$ arsenic were analyzed every 10 samples as calibration check solutions.

Adam N, Schmitt C, Galceran J, Companys E, Vakurov A, Wallace R, Knapen D, Blust R (2014): The chronic toxicity of $\mathrm{ZnO}$ nanoparticles and $\mathrm{ZnCl} 2$ to Daphnia magna and the use of different methods to assess nanoparticle aggregation and dissolution. Nanotoxicology 8, 709-717 


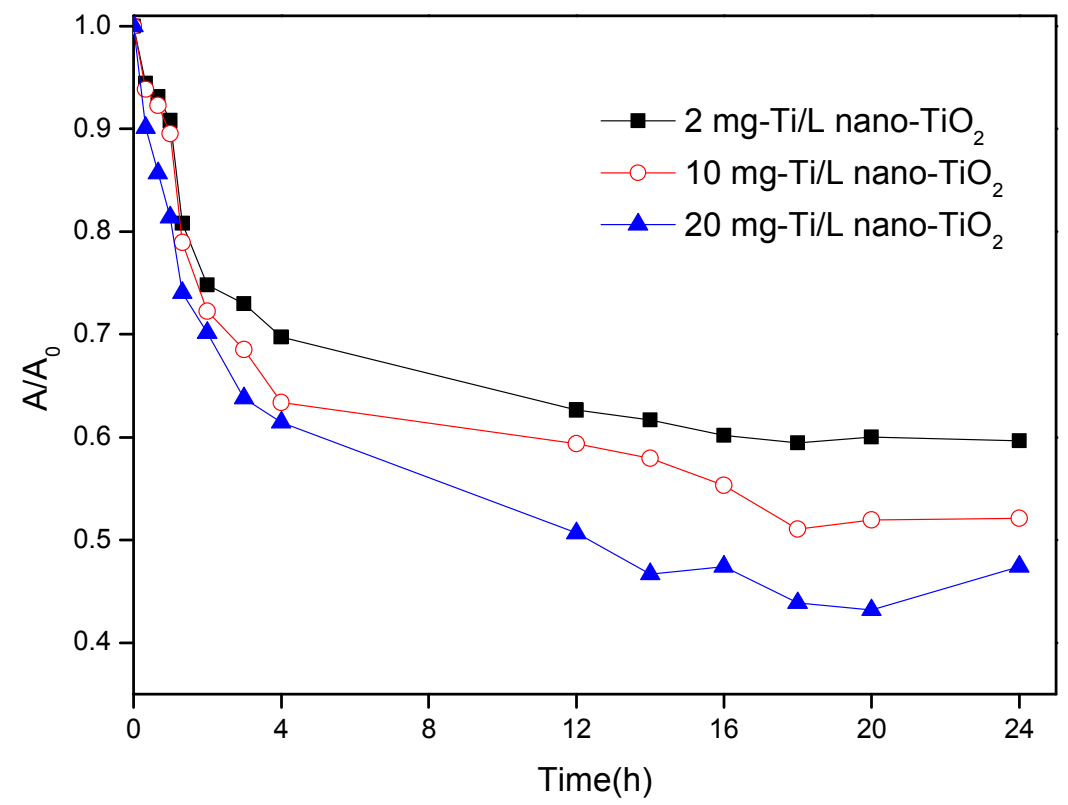

Fig.S1. Sedimentation of nano-TiO2 under different concentration in SM7. 
Table S1. Typical operation conditions for LA-ICP-MS analysis

\begin{tabular}{ll}
\hline ICP-MS conditions & \\
RF power & $1350 \mathrm{~W}$ \\
Plasma gas & $14 \mathrm{~L} \mathrm{~min}^{-1} \mathrm{Ar}$ \\
Auxiliary gas & $0.9 \mathrm{~L} \mathrm{~min}^{-1} \mathrm{Ar}$ \\
Make-up gas & $0.8 \mathrm{~L} \mathrm{~min}^{-1} \mathrm{Ar}$ \\
Sampling depth & $5.4 \mathrm{~mm}$ \\
Detector & Dual (pulse and analog counting) \\
Dwell time/mass & $6 \mathrm{~ms}$ \\
Laser parameters & \\
Wavelength & $193 \mathrm{~nm}$ \\
Energy density & $14 \mathrm{~J} \mathrm{~cm}{ }^{-2}$ \\
Carrier gas & $\mathrm{He}($ optimized to get the highest sensitivity) \\
Ablation style & Single spot \\
Ablation spot size & $32 \mu \mathrm{m}$ \\
Repetition rate & $8 \mathrm{~Hz}$ \\
Laser pulse & 480 \\
\hline
\end{tabular}




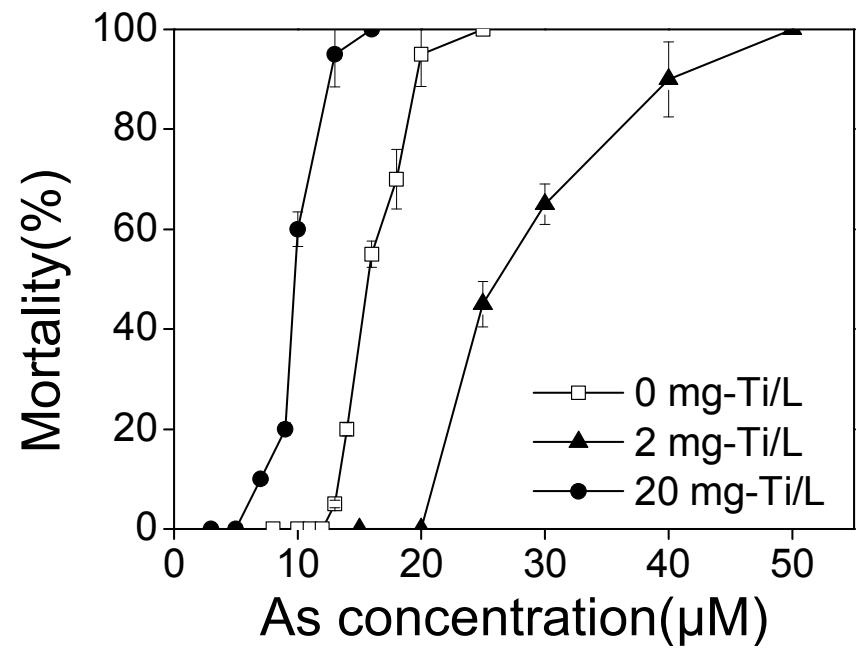

Fig. S2. Toxic effect of $\mathrm{As}(\mathrm{V})$ in the presence of nano- $\mathrm{TiO}_{2}$. 

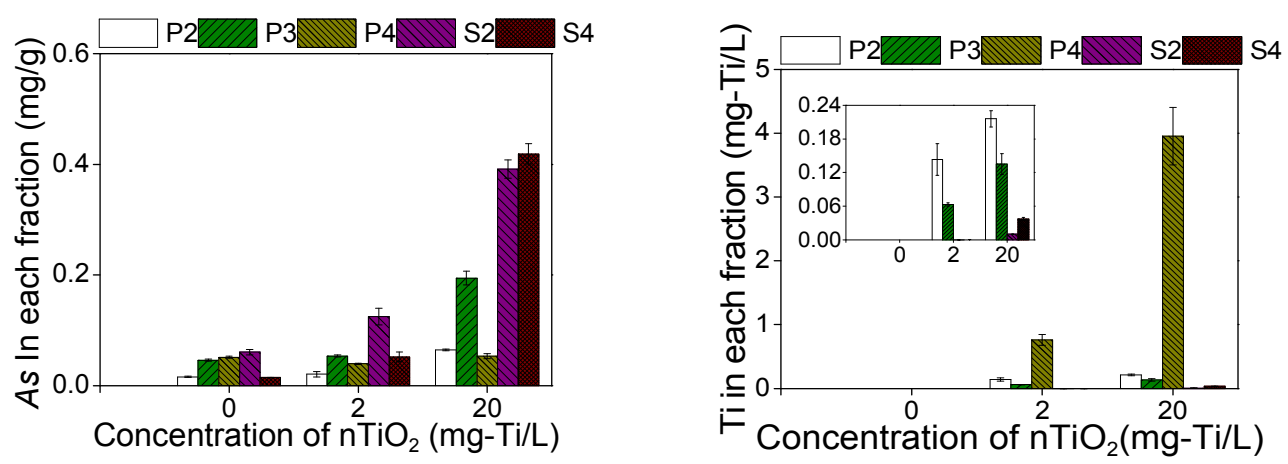

Fig. S3. Subcellular distribution of total arsenic and Ti in Daphnia magna after exposure to different nano- $\mathrm{TiO}_{2}$ concentrations for $3 \mathrm{~h}$. Mean \pm standard deviation $(n=3)$. P2- Metal-Rich Granules. P3- Organelles. P4- Heat-Sensitive Protein. S2Cellular Debris. S4- Heat-Denatured Protein. 